\title{
Retrocesso do Programa de Controle da Esquistossomose no estado de maior prevalência da doença no Brasil
}

\section{Setback of the Schistosomiasis Control Program in the Brazilian state with the highest prevalence of the disease}

\author{
José Icaro Nunes Cruz' (D), Gabriela de Oliveira Salazar1 (D), Roseli La Corte ${ }^{2}$ (D) \\ 1 Universidade Federal de Sergipe, Centro de Ciências Biológicas e da Saúde, Departamento de Medicina, Aracaju, Sergipe, Brasil \\ 2 Universidade Federal de Sergipe, Centro de Ciências Biológicas e da Saúde, Departamento de Morfologia, Aracaju, Sergipe, Brasil
}

\begin{abstract}
RESUMO
OBJETIVO: Descrever o quadro epidemiológico da esquistossomose no contexto do Programa de Controle da Esquistossomose (PCE) no estado de Sergipe, Brasil. MATERIAIS E MÉTODOS: Foi desenvolvido um estudo ecológico de séries temporais, baseado em dados secundários do Sistema de Informação do Programa de Controle da Esquistossomose da Secretária Estadual de Saúde de Sergipe, de 2008 a 2017. As informações extraídas foram analisadas com os programas TabWin, Joinpoint Regression e BioEstat. RESULTADOS: Em média, 36,7 \pm 5,6 municípios participaram do PCE no período, com o total de 677.841 exames realizados, dos quais 59.996 foram positivos (média anual de 8,6\% 1 1,2\%); 4.566 dos casos positivos apresentaram alta carga parasitária (média de 7,9\% $\pm 2,1 \%$ ). De todos os casos positivos, 42.779 foram tratados (média de 68,7\% $\pm 9,5 \%$ ). O percentual de casos com alta carga parasitária apresentou tendência crescente estatisticamente significante (5,7\% ao ano). Apresentaram tendência decrescente estatisticamente significante a adesão de municípios ao PCE $(4,0 \%$ por ano) e o número de exames realizados $(9,6 \%$ por ano). Em relação à distribuição espacial da esquistossomose, em 2011, ano de maior adesão de municípios ao PCE, cinco, 30 e 10 municípios foram, respectivamente, considerados de alta, média e baixa endemicidade. Em 2017, Siriri foi considerado como de alta endemicidade, 20 municípios foram de média endemicidade e cinco de baixa endemicidade. CONCLUSÃO: As ações do PCE diminuíram em Sergipe, o que somado à aparente subnotificação e ao aumento de casos com alta carga parasitária, compromete os ganhos obtidos no controle da doença.
\end{abstract}

Palavras-chave: Esquistossomose; Schistosoma mansoni; Saúde Pública; Epidemiologia; Doenças Negligenciadas.

\section{ABSTRACT}

OBJECTIVE: To describe the epidemiological picture of schistosomiasis in the context of the Schistosomiasis Control Program (SCP) in Sergipe State, Brazil. MATERIALS AND METHODS: An ecological time series study was conducted based on secondary data from the Information System of the Schistosomiasis Control Program of the State Secretary of Health of Sergipe, 2008-2017. Data were analyzed using TabWin, Joinpoint Regression, and BioEstat. RESULTS: On average, $36.7 \pm 5.6$ municipalities participated in the SCP, with 677,841 exams performed, of which 59,996 were positive (annual average of $8.6 \% \pm 1.2 \%) ; 4,566$ of the positive cases had a high parasitic burden $(7.9 \% \pm 2.1 \%)$. Of all positive cases, 42,779 were treated $(68.7 \% \pm 9.5 \%)$. The percentage of cases with a high parasitic load showed a statistically significant increasing trend (5.7\% per year). There was a statistically significant downward trend in the number of municipalities joining the SCP ( $4.0 \%$ per year) and the number of tests performed $(9.6 \%$ per year). Regarding the spatial distribution of schistosomiasis, in 2011, the year with the highest number of municipalities adhering to the SCP, five, 30, and 10 municipalities were considered, respectively, of high, medium, and low endemicity. In 2017, Siriri was considered to be highly endemic, 20 municipalities were of medium endemicity, and five of low endemicity. CONCLUSION: The actions of the SCP decreased in Sergipe, in addition to the apparent underreporting of cases and the increase in cases with a high parasitic burden, compromising the gains obtained in the control of the disease.

Keywords: Schistosomiasis; Schistosoma mansoni; Public Health; Epidemiology; Neglected Diseases.

\section{Correspondência / Correspondence:}

Roseli La Corte

Universidade Federal de Sergipe, Laboratório de Entomologia e Parasitologia Tropical

Av. Marechal Rondon, s/n. Bairro: Rosa Elze. CEP: 49100-000 - São Cristóvão, Sergipe, Brasil - Tel.: +55 (79) $3194-6626$

E-mail: rlacorte@ufs.br 


\section{INTRODUÇÃO}

A esquistossomose está entre as doenças parasitárias de maior prevalência mundial'. De acordo com relatórios da Organização Mundial da Saúde (OMS), essa parasitose pertence ao grupo de doenças tropicais negligenciadas, com quase 240 milhões de pessoas no mundo necessitando de tratamento ${ }^{2}$. Em 2018, foram tratadas mais de 97,2 milhões de pessoas ${ }^{3}$

A esquistossomose foi registrada em 78 países, incluindo sobretudo regiões das Américas, África e Mediterrâneo Oriental. Nas Américas, o Brasil é o país mais afetado, com cerca de 1,5 milhão de pessoas infectadas por Schistosoma mansoni e mais de 25 milhões habitando locais com alto risco de infecção $3,4,5,6,7$. A transmissão autóctone foi relatada em todas as cinco regiões brasileiras, sendo os estados endêmicos Alagoas, Bahia, Maranhão, Paraíba, Pernambuco, Rio Grande do Norte, Sergipe, Espírito Santo e Minas Gerais ${ }^{4}$. Atualmente, a esquistossomose é encontrada em uma extensão contínua que envolve - Rio Grande do Norte até o norte de Minas Gerais, adentrando o interior da Bahia, Minas Gerais e Maranhão. Nos estados das Regiões Norte, Centro Oeste e Sul, a transmissão ocorre em áreas restritas, sendo considerada focal ${ }^{8}$.

Diante da expansão da doença no país, em 1975 foi criado o Programa Especial de Controle da Esquistossomose (PECE), que visou eliminar a transmissão e reduzir a prevalência da infecção para menos de 4\%. Apesar dos bons resultados, o objetivo principal não foi alcançado. Posteriormente, - PECE foi substituído pelo Programa de Controle da Esquistossomose (PCE) na década de 19809,10. Em 1993, houve a descentralização das ações da vigilância e controle da esquistossomose pela criação do Projeto de Controle de Doenças Endêmicas no Nordeste, aumentando a participação dos municípios e também a cobertura do PCE. Atualmente, o controle da doença depende da implementação de políticas públicas, sob responsabilidade dos gestores municipais e está inserido entre as atividades da Atenção Básica ${ }^{5}$.

A implementação de medidas de controle da esquistossomose, desde 1976, ocasionou redução das formas hepatoesplênicas e do número de óbitos. Após essa melhoria, a doença atingiu um novo estado endêmico, com resposta insatisfatória às ações de controle ${ }^{5}$. Em 2010, o Inquérito Nacional da Prevalência da Esquistossomose mansoni e Geo-Helmintoses (INPEG) evidenciou queda significativa nas taxas de positividade em todos os estados brasileiros, com os maiores índices de prevalência nas Regiões Norte e Nordeste, tendo o estado de Sergipe apresentado a maior proporção de positivos (8,19\%), muito superior ao observado para a Região Nordeste $(1,27 \%)^{11}$. Desse modo, Sergipe apresenta, historicamente, alta prevalência para essa parasitose, com registros de prevalência superior a $50 \%$ em municípios do leste do estado ${ }^{12,13}$

Em virtude das dificuldades para a vigilância e controle da esquistossomose, é importante compreender a dinâmica da transmissão e os fatores a ela associados $^{14}$. A transmissão da doença ocorre por veiculação hídrica em corpos de água contaminados com esgoto doméstico. Esse modo de infecção explica sua maior prevalência na população de baixa renda, em destaque as comunidades agrícolas e pesqueiras, estando, portanto, associada a baixas condições socioeconômicas e tendo como condicionantes um saneamento básico deficiente, más condições de higiene, ausência de educação voltada para a saúde e contato frequente do homem com águas contaminadas por esgotos domésticos 3,11 .

Além de afetar a saúde da população, a esquistossomose causa importantes impactos econômicos ${ }^{15}$. Primeiramente, essa parasitose é mais incapacitante que letal ${ }^{3,16}$, reduzindo o número da população economicamente ativa. Além disso, a esquistossomose gera altos custos à saúde pública, divididos entre os custos diretos (diagnóstico e tratamento de complicações), custos diretos não relacionados à saúde (transporte e atendimento doméstico) e custos indiretos (auxílio doença e morte prematura) $)^{17}$. O turismo, por sua vez, também é afetado, visto que alguns pontos turísticos apresentam potencial risco de infecção ${ }^{18}$

As contribuições científicas para o conhecimento da esquistossomose foram muito significativas. Entretanto, ainda há escassez de estudos recentes que desenhem a condição local dessa doença e analisem o funcionamento e os resultados das medidas de controle. Nesse contexto, e considerando que Sergipe é o estado de maior prevalência dessa parasitose no Brasill1, este estudo teve por objetivo descrever o quadro epidemiológico da esquistossomose no estado e avaliar as atividades relacionadas ao PCE em análise de série temporal.

\section{MATERIAIS E MÉTODOS}

Este estudo analisou dados da esquistossomose mansoni em Sergipe, o menor estado brasileiro em território $\left(21.926,908 \mathrm{~km}^{2}\right)$, localizado na Região Nordeste. Sua população é composta por 2.068.017 habitantes, e a densidade demográfica é de 94,36 habitantes $/ \mathrm{km}^{2}$. Sergipe é dividido em 75 municípios, sendo a capital Aracaju localizada na região litoral do estado ${ }^{19}$.

Para a análise dos aspectos epidemiológicos da esquistossomose mansoni, foi realizado um estudo ecológico de séries temporais de 2008 a 2017 . Foram utilizados dados secundários do Sistema de Informação do Programa de Controle da Esquistossomose (SISPCE) da Secretaria de Estado da Saúde de Sergipe (SES/SE) e dados disponíveis no Departamento de Informática do Sistema Único de Saúde (DATASUS). Fizeram parte dos dados coletados pelo PCE somente os municípios considerados endêmicos para essa parasitose (51 municípios) ${ }^{20}$. No período de 2008 a 2011, os dados disponíveis no DATASUS 6 foram semelhantes aos dados da SES/SE. No entanto, a partir de 2012, os dados do DATASUS mostraram-se significativamente menores e incompletos. Portanto, para os anos de 2012 a 2017 , foram utilizadas as informações disponíveis em planilhas oficiais da SES/SE. 
Foram compilados dados anuais, por município, referentes às seguintes informações: a) população trabalhada; b) número de exames realizados; c) número de positivos para $S$. mansoni; d) percentual de positividade para S. mansoni; e) número de positivos por carga parasitária; f) número de indivíduos a serem tratados para S. mansoni; e g) número de indivíduos tratados para S. mansoni. Considerou-se adesão ao PCE o registro de atividades do município no SISPCE em cada ano.

Os dados foram tabulados no programa Microsoft Excel $2016^{\circledR 21}$ e posteriormente calculados, por ano: número de exame realizados; percentual de adesão dos municípios endêmicos ao PCE (considerando o total de 51 municípios endêmicos); percentual de positividade da população avaliada; percentual de casos com alta carga parasitária (17 ou mais ovos por lâmina equivalente a aproximadamente 400 ovos ou mais por grama de fezes ${ }^{22}$ ); e percentual de tratados para esquistossomose mansoni. $\bigcirc$ método utilizado para a realização dos exames parasitológicos de fezes foi o Kato-Katz, o preconizado pelo Ministério da Saúde para o PCE 5,11 .

Os resultados foram analisados por ano e como média de todo o período, associada ao desvio padrão. Para as variáveis percentual de positividade, percentual de positivos com alta carga parasitária e percentual de tratados, foi necessário realizar cálculo de média ponderada, utilizando como pesos, respectivamente, o número de exames realizados, o número de casos positivos e o número de pessoas a tratar. Abaixo descreve-se o cálculo realizado, onde "P" corresponde ao percentual por ano e "Pe" ao peso:

Média Ponderada $=\frac{\left(P_{2008}{ }^{*} P e_{2008}\right)+\left(P_{2009}{ }^{*} P e_{2009}\right)+\cdots+\left(P_{2017}{ }^{*} P e_{2017}\right)}{P e_{2008}+P e_{2009}+\cdots+P e_{2017}}$
Utilizou-se o programa Joinpoint Regression v4.7.0.0 ${ }^{\circledR 23}$ para a construção de gráficos de dispersão, relacionando cada uma das variáveis citadas (dependentes) ao ano (variável independente), com demonstração da variação anual percentual (annual percentage change - APC), considerando o intervalo de confiança $(\mathrm{Cl})$ de $95 \%$. A regressão linear foi determinada pela regressão de Poisson e a correlação entre as variáveis pela correlação de Spearman, expressa pelo coeficiente de correlação de Spearman (rs), utilizando o programa BioEstat v5. $3^{\circledR 24}$.

Por fim, avaliou-se a distribuição espacial da esquistossomose no estado de Sergipe em relação ao percentual de positividade nos anos de maior e menor adesão dos municípios endêmicos ao PCE (2011 e 2017, respectivamente), por meio da construção de mapas temáticos, demonstrando o percentual de positividade da esquistossomose mansoni por município. A elaboração dos mapas foi realizada pelo programa TabWin v4.1.56. Os municípios tiveram seus percentuais de positividade distribuídos em cinco intervalos: I) 0 a 9\%; II) 10 a 18\%; III) 19 a 27\%; IV) 28 a 36\%; e V) 37 a 45\%. Com base nas definições contidas no INPEG ${ }^{11}$, os municípios com percentual de positividade abaixo de 5\% foram considerados de baixa endemicidade; entre 5\% e 25\%, de média endemicidade; e acima de $25 \%$, de alta endemicidade.

\section{RESULTADOS}

Entre 2008 e 2017, a adesão média dos municípios endêmicos de Sergipe ao PCE foi $72 \% \pm 11 \%$ $(36,7 \pm 5,6)$, sendo a maior adesão $88,2 \%$ (45) em 2011 e a menor $51 \%$ (26) em 2017. Foram realizados 677.861 exames durante o período estudado, com média anual de $67.786 \pm 23.808$. $\bigcirc$ maior número de exames realizados foi 100.387 em 2011 e o menor foi 30.494 em 2016 (Tabela 1).

Tabela 1 - Série histórica com a distribuição de exames realizados e de percentuais de adesão dos municípios ao PCE, positividade para S. mansoni, positivos com alta carga parasitária e tratamento realizado no estado de Sergipe (2008-2017)

\begin{tabular}{|c|c|c|c|c|c|}
\hline \multirow[b]{2}{*}{ Ano } & \multicolumn{5}{|c|}{ Variáveis } \\
\hline & $\begin{array}{l}\text { Número de exames } \\
\text { realizados }\end{array}$ & $\begin{array}{c}\text { Percentual de } \\
\text { adesão de } \\
\text { municípios ao PCE }\end{array}$ & $\begin{array}{l}\text { Percentual de } \\
\text { positividade para } \\
\text { S. mansoni }\end{array}$ & $\begin{array}{l}\text { Percentual de } \\
\text { positivos com alta } \\
\text { carga parasitária }\end{array}$ & $\begin{array}{l}\text { Percentual de } \\
\text { tratados para } \\
\text { S. mansoni }\end{array}$ \\
\hline 2008 & 79.858 & 76,5 & 10,3 & 6,7 & 77,5 \\
\hline 2009 & 92.957 & 80,4 & 8,8 & 5,4 & 52,3 \\
\hline 2010 & 91.719 & 82,3 & 9,6 & 7,5 & 64,1 \\
\hline 2011 & 100.387 & 88,2 & 8,5 & 7,9 & 75,2 \\
\hline 2012 & 71.585 & 76,5 & 7,6 & 6,9 & 65,0 \\
\hline 2013 & 62.542 & 64,7 & 8,1 & 11,9 & 78,5 \\
\hline 2014 & 47.058 & 64,7 & 6,9 & 8,0 & 79,0 \\
\hline 2015 & 61.485 & 70,6 & 7,4 & 8,6 & 77,0 \\
\hline 2016 & 30.494 & 64,7 & 9,9 & 11,7 & 58,3 \\
\hline 2017 & 39.776 & 51,0 & 7,1 & 8,8 & 73,3 \\
\hline Média do período total & 67.786 & 72,0 & $8,6^{*}$ & $7,9^{*}$ & $68,7^{*}$ \\
\hline
\end{tabular}

* Média ponderada de acordo com o número de exames realizados, número de casos positivos e número de pessoas a tratar em cada ano. 
A positividade média para S. mansoni nos exames realizados foi de $8,6 \% \pm 1,2 \%(5.799 \pm 2.448$ exames positivos), sendo o maior percentual 10,3\% (8.258) em 2008, e o menor 6,9\% (3.227) em 2014 (Tabela 1). A capital, Aracaju, demonstrou média de positividade inferior às médias para o estado ao longo dos anos $(2,8 \% \pm 1,2 \%$ ao ano), sendo 2010 o ano de maior positividade $(4,6 \%)$.

Dentre o número total de positivos, observou-se média de 7,9\% $\pm 2,1 \%(457 \pm 157)$ casos com alta carga parasitária por ano, atingindo o valor máximo de $11,9 \%$ (604) em 2013 e valor mínimo de 5,4\% (442) em 2009. Em relação à cobertura de tratamento, registrou-se proporção média de 68,7\% \pm 9,5\% (4.278 \pm 1.606$)$ pacientes tratados por ano, variando de 58,3\% (2.618) em 2016 a 79,0\% (2.567) em 2014 (Tabela 1).

Ao longo da série temporal estudada, o percentual de adesão dos municípios sergipanos ao PCE apresentou tendência de redução estatisticamente significante de aproximadamente $4 \%$ ao ano (APC $=-3,96 ; \mathrm{Cl}=-1,4 \mathrm{a}-6,4 ; \mathrm{p}=0,0007$ ) (Figura 1). $\mathrm{O}$ número de exames realizados pelo Programa também sofreu redução estatisticamente significativa de $9,6 \%$ ao ano ( $\mathrm{APC}=-9,58 ; \mathrm{Cl}=-14,4$ a -4,4; $\mathrm{p}=0,0031$ ), o que demonstra queda na cobertura populacional dos inquéritos coproscópicos do PCE (Figura 2).

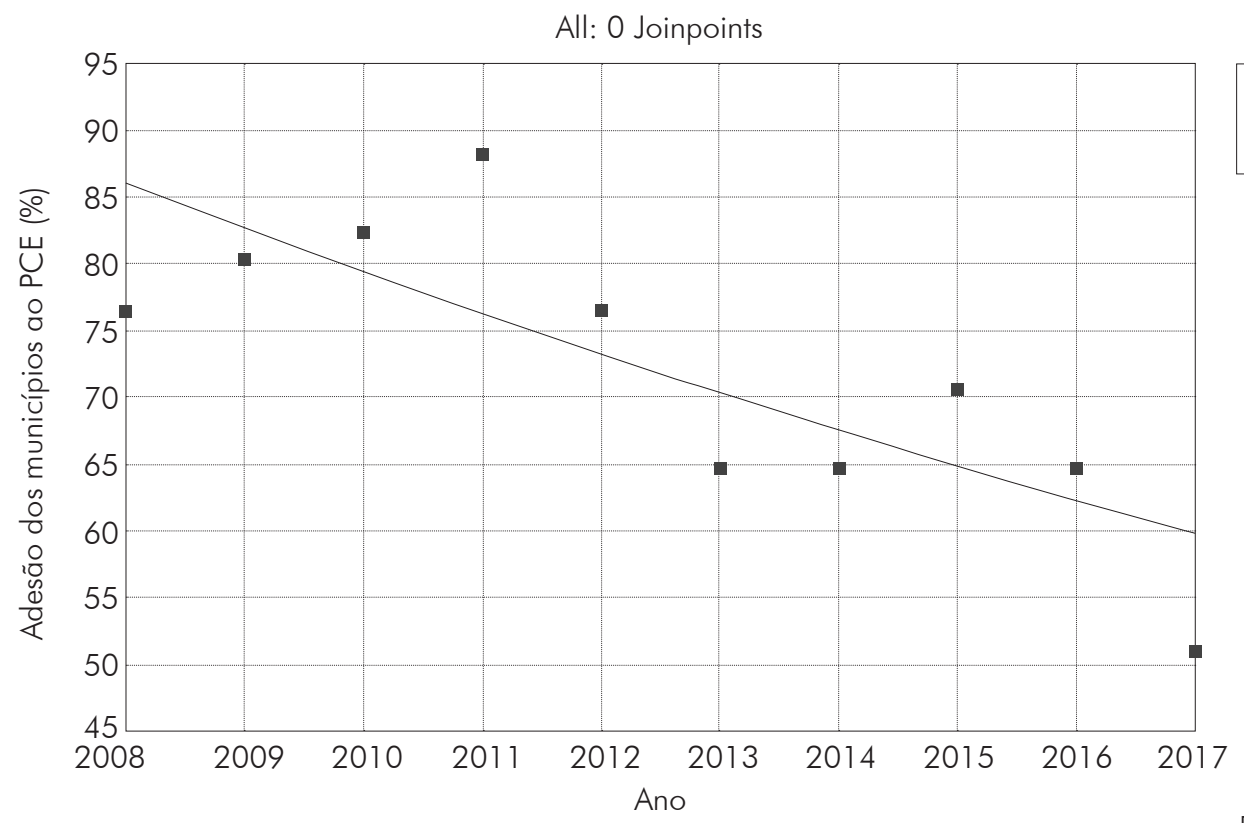

Observed

2008.0-2017.0 APC $=-3.96^{*}$

Fonte: SISPCE-SE/SES-SE/DATASUS, 2019 * Indicates that the Annual Percent Change (APC) is significantly different from zero at the alpha $=0.05$ level. Final Selected Model: 0 Joinpoints.

Figura 1 - Distribuição, por ano, da proporção de adesão dos municípios sergipanos ao PCE (2008-2017)

All: 0 Joinpoints

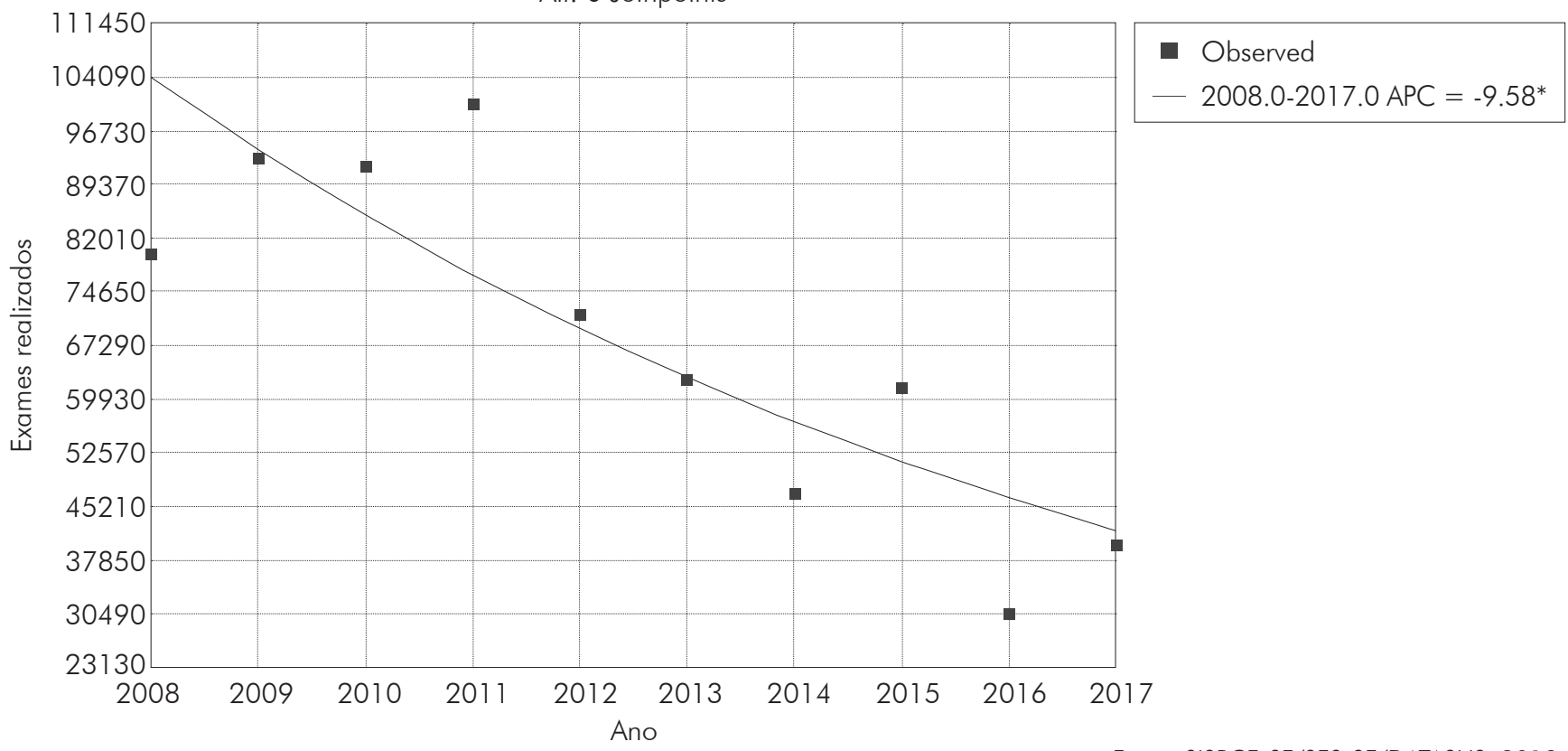

Fonte: SISPCE-SE/SES-SE/DATASUS, 2019.

* Indicates that the Annual Percent Change (APC) is significantly different from zero at the alpha $=0.05$ level. Final Selected Model: 0 Joinpoints.

Figura 2 - Número de exames realizados para S. mansoni, por ano, no estado de Sergipe (2008-2017) 
A evolução do percentual de pacientes com alta carga parasitária para S. mansoni apresentou tendência crescente, com aumento percentual estatisticamente significante de $5,7 \%$ ao ano $(A P C=5,67 ; \mathrm{Cl}=0,4$ a 11,$3 ; p=0,038$ ) (Figura 3).

A análise de regressão linear para as seguintes variáveis não demonstrou significância estatística: percentual de positividade para S. mansoni (APC $=-2,49 ; \mathrm{Cl}=-5,6$ a 0,$7 ; \mathrm{p}=0,1)$; e percentual de pacientes tratados $(\mathrm{APC}=0,73 ; \mathrm{Cl}=-2,8$ a 4,4 ; $p=0,7)$.

Houve correlação negativa estatisticamente significante entre o percentual de adesão de municípios ao PCE e o percentual de pacientes com alta carga parasitária ( $r s=-0,7139 ; p=0,0203$ ) e também entre o número de exames realizados anualmente e - percentual de pacientes com alta carga parasitária $(r s=-0,7091 ; p=0,0216)$.

A distribuição espacial da esquistossomose mansoni em Sergipe foi observada em termos de percentual de positividade (Figura 4). Foram avaliados os anos de 2011 e 2017, em que houve a maior (45) e menor adesão (26) de municípios ao PCE, respectivamente. Em 2011, foram realizados 100.387 exames e o percentual de positividade médio foi de $8,5 \%$, dos quais $75,2 \%$ foram tratados. Nesse ano, os seguintes municípios apresentaram alta endemicidade: Cristinápolis (25,2\%), Japoatã $(27,1 \%)$, Maruim $(28,8 \%)$, Santa Rosa de Lima $(30,5 \%)$ e São Cristóvão (32,8\%). Além desses cinco, 30 municípios mostraram média endemicidade e 10 municípios foram considerados de baixa endemicidade, dentre os quais está a capital do estado, Aracaju (4,4\%). Em 2017, foram realizados 39.776 exames, e o percentual de positividade médio foi de 7,1\%, dos quais $73,3 \%$ foram tratados. Nesse ano, o município Siriri apresentou prevalência de 25,5\%, sendo o único município de alta endemicidade. Apresentaram média endemicidade 20 municípios e cinco mostraram baixa endemicidade, incluindo Aracaju $(1,4 \%)$.

\section{DISCUSSÃO}

Sergipe foi apontado como o estado brasileiro de maior prevalência de esquistossomose no último inquérito realizado no Brasil ${ }^{11}$. Entretanto, apesar dessa constatação, os resultados da presente análise apontaram redução da atenção à esquistossomose. $\mathrm{O}$ número de municípios executando as atividades do PCE, de 2008 a 2017, apresentou tendência decrescente e redução aproximada de $4 \%$ ao ano, de modo que, em 2017, somente 26 municípios registraram atividades, excluindo, inclusive, municípios considerados de alta endemicidade em períodos anteriores, como Japoatã, Maruim e Cristinápolis. Essa queda na adesão ao Programa traz sérias consequências para o estado, uma vez que a Secretária de Vigilância em Saúde (SVS) fornece insumos e apoios técnico e financeiro baseados em relatórios estatísticos e análises do PCE advindos das secretarias estaduais e municipais de Saúde ${ }^{25}$. Dado relevante é que essa redução de adesão ao PCE coincide com a emergência - ou agravamento - de outras epidemias no estado, como dengue, chikungunya, zika, dentre outras, sugerindo possível desvio da atenção dos órgãos de saúde pública $26,27,28,29,30,31$.

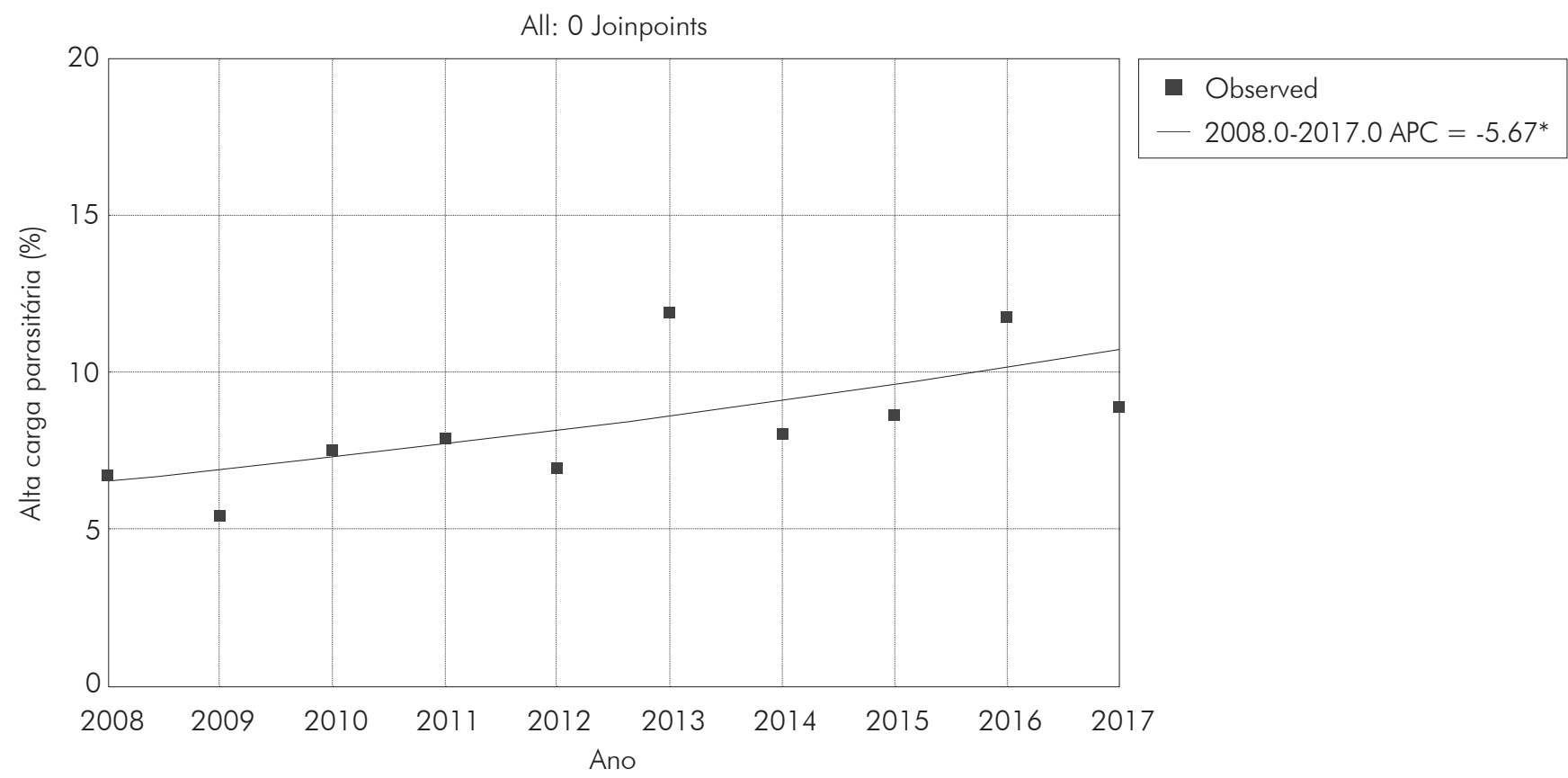

Fonte: SISPCE-SE/SES-SE/DATASUS, 2019. * Indicates that the Annual Percent Change (APC) is significantly different from zero at the alpha $=0.05$ level. Final Selected Model: 0 Joinpoints.

Figura 3 - Distribuição da frequência percentual de positividade para S. mansoni com alta carga parasitária no estado de Sergipe (2008-2017) 

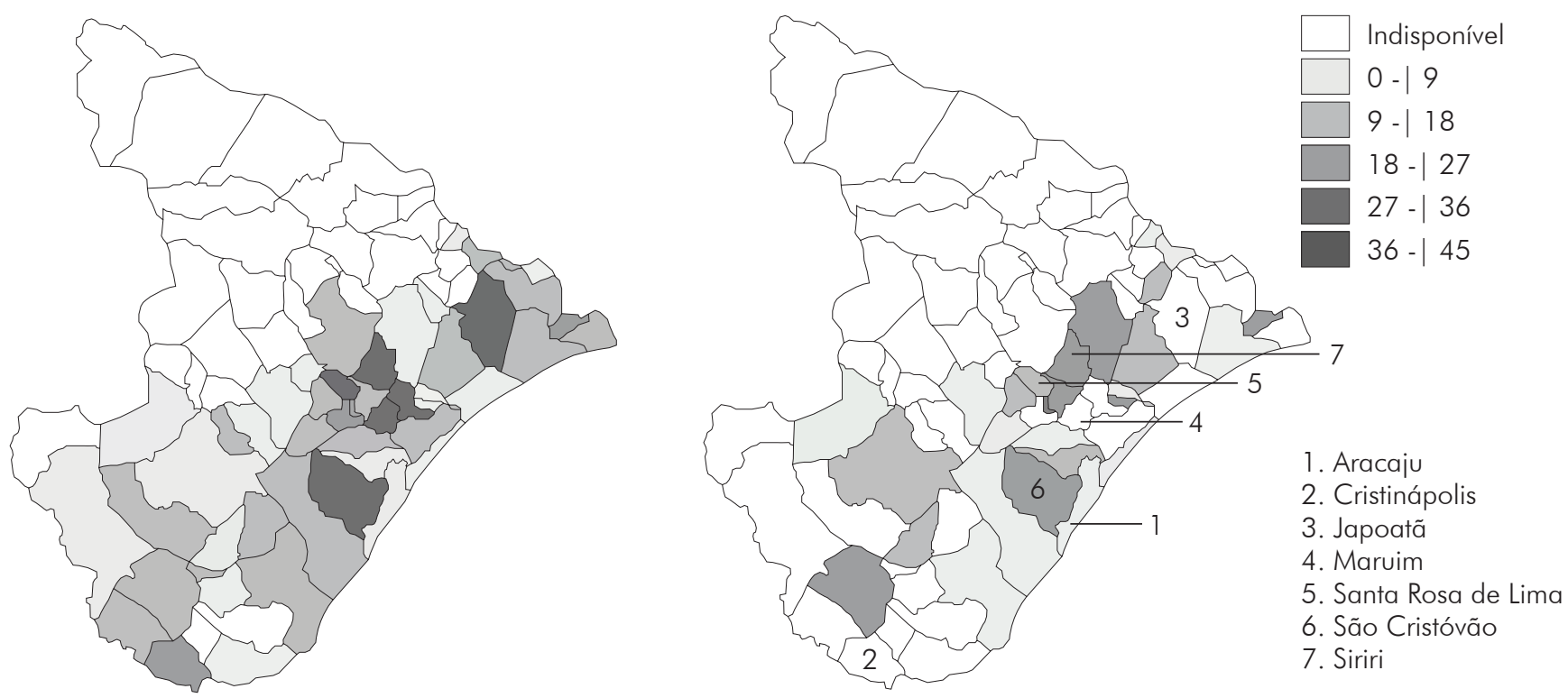

A: 2011 , ano de maior adesão dos municípios ao PCE; B: 2017, ano de menor adesão dos municípios ao PCE.

Figura 4 - Mapa do percentual de positividade para S. mansoni no estado de Sergipe, em 2011 e 2017

Em decorrência do menor número de municípios endêmicos realizando atividades do Programa, o número de exames realizados diminuiu, apresentando tendência de queda de aproximadamente 9,6\% por ano, o que assinala provável subnotificação de casos. Outros autores também relataram essa redução no número de exames realizados em períodos semelhantes ao estudado ${ }^{26,32}$, paralelo a decréscimo da adesão de municípios ao PCE ${ }^{25}$. Esses fenômenos ocorreram pouco após a descentralização das ações de controle da endemia e podem gerar dados errôneos acerca da distribuição da doença no estado, bem como interpretações equivocadas acerca do real cenário da doença ${ }^{25}$. Soma-se a isso, a discordância de informações, a partir de 2012, entre o DATASUS e os dados da SES/SE que, além de contribuir para tais erros de interpretação, apontam falhas no fluxo de registro das notificações entre municípios, estados e União.

A proporção de exames positivos para esquistossomose apresentou oscilações durante 0 período estudado, com média de $8,6 \%$. Em 2011, ano de maior adesão dos municípios, a positividade foi de $8,5 \%$, percentual semelhante à média dos 10 anos e superior à média nordestina no mesmo $\mathrm{ano}^{33}$, bem como a de outros estados de alta prevalência da doença, como Alagoas $^{34}$. Embora a positividade não expresse a prevalência da doença, uma vez que os inquéritos coproscópicos não são realizados para este fim e não têm base em amostra representativa da população, a média encontrada é também semelhante à prevalência obtida no inquérito nacional $(8,19 \%)$ realizado entre 2010 e 201511. De fato, Sergipe é um estado historicamente endêmico, com condições favoráveis ao estabelecimento

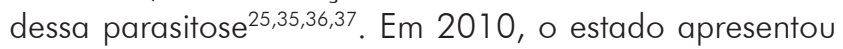
déficit de saneamento básico, com quase o dobro do percentual de pessoas vivendo em domićlios com serviços de abastecimento de água e esgotamento sanitário inadequados $(11 \%)$ em relação ao país $(6 \%)^{38}$.
Referente à prevalência municipal no período mais recente avaliado (2017), destacam-se llha das Flores, Itabaianinha, São Cristóvão e Siriri, que registraram taxa de positividade superior a $20 \%$. Estes municípios apresentaram Índices de Desenvolvimento Humano Municipal (IDHM) considerados médios em 2010 $(0,562 ; 0,556 ; 0,662 ;$ e 0,609, respectivamente) e notavelmente inferiores ao IDHM nacional $(0,727)^{39}$. A capital sergipana, de IDHM alto $(0,770)^{38}$, contudo, apresentou positividade de 4,6\% no mesmo ano. Essa comparação pode indicar uma relação entre o grau de desenvolvimento humano e a prevalência da esquistossomose, desde que presentes os elos da cadeia de transmissão ${ }^{40}$. Além das baixas condições socioeconômicas e do déficit na infraestrutura serem fatores determinantes da alta prevalência da esquistossomose ${ }^{26}$, a heterogeneidade e a irregularidade na execução de programas de controle ${ }^{41}$ também devem ser reconhecidos como contribuintes para a desigualdade da positividade entre os municípios. Em 2017, por exemplo, vários municípios considerados de elevada endemicidade não registraram ações do PCE (Figura 4).

Outro fato importante é a modificação na dinâmica de transmissão da doença em resposta às modificações ambientais provocadas pelo homem. Sabe-se que, com a urbanização, grandes áreas do solo são impermeabilizadas, o que dificulta a drenagem da água da chuva e possibilita a formação de alagados muitas vezes contaminados com efluentes domésticos. Diante disso, a transmissão da esquistossomose passou a ser não somente associada a lagoas e outros reservatórios em áreas rurais, mas também a alagadiços peridomiciliares nas áreas urbanas, com aumento de casos autóctones ${ }^{42,43,44,45}$. Esse mecanismo de transmissão pode ser evitado após implantação de medidas para adequada drenagem de águas pluviais e tratamento dos esgotos ${ }^{46}$. 
No que diz respeito à gravidade da infecção, este estudo expôs um aumento na carga parasitária, passando de 5,4\% (2009) para 11,9\% (2013), com valores mantendo-se superiores a 8\% desde 2013, em contramão com a redução histórica observada em outros estados brasileiros após a adoção dos programas de controle ${ }^{34,47}$. $\bigcirc$ aumento da carga parasitária mostrou correlação com a redução das atividades do Programa e do número de exames realizados. Esse encontro pode ser resultado do enfraquecimento das ações para o diagnóstico precoce, embora se deva considerar a possibilidade de viés referente à não adesão de municípios que poderiam apresentar menores percentuais de alta carga parasitária. Porém, municípios de média e elevada endemicidade também estão deixando de realizar as atividades do PCE (Figura 4). A observação do aumento da carga parasitária é muito relevante, uma vez que a intensidade da infecção possui relação direta com a gravidade da doença, com consequências graves para o paciente e aumento do custo para os serviços de sáude ${ }^{48}$. Além disso, a cobertura de tratamento apresentou média de 68,7\% $\pm 9,5 \%$, percentual ainda inferior à meta de $80,0 \%$ de tratamento definida pelo PCE ${ }^{5}$. Esse déficit pode refletir falhas nos planejamentos das ações, como busca ativa de pacientes em horários incompatíveis com a sua rotina, ou a dificuldade do acesso do doente aos serviços de saúde da atenção primária ${ }^{32}$.

Em 2012, o Ministério da Saúde divulgou o "Plano integrado de ações estratégicas de eliminação da hanseníase, filariose, esquistossomose e oncocercose como problema de saúde pública, tracoma como causa de cegueira e controle das geo-helmintíases" em que, dentre seus objetivos, visava eliminar a esquistossomose como problema de saúde pública nos municípios endêmicos até 2015. Um dos objetivos desse plano era realizar a cobertura de $100 \%$ de tratamento de casos positivos $^{16}$. Como esta análise demonstra, além de não atingir as metas programadas, houve retrocesso no estado de Sergipe, situação que também foi reproduzida em outros estados brasileiros ${ }^{34}$.

Este estudo apresenta como limitação o uso de dados secundários, gerados a partir da execução e registro dos dados do PCE, que pode ter sido diferente em cada município, como a abrangência e a frequência dos inquéritos e a inserção na atenção básica. No entanto, ele é útil no que tange ao reconhecimento do cenário de possível perda de prioridade do PCE em Sergipe. Somente com a retomada da implementação do programa de controle e a execução de novas medidas junto com a efetivação do Plano Nacional de Saneamento Básico ${ }^{49}$, - Brasil pode se aproximar da meta de eliminação da esquistossomose como um problema de saúde pública até 2025, definida pela $\mathrm{OMS}^{3}$.

\section{CONCLUSÃO}

Conforme análise dos dados, houve diminuição das atividades do PCE nos municípios sergipanos durante o período de 2008 a 2017. Além disso, observou-se tendência de aumento de casos com alta carga parasitária e, provavelmente, de casos graves, com consequente aumento de custos em saúde pública ao estado. Em nenhum ano foi atingida a meta de $80 \%$ de cobertura de tratamento definida pelo Programa. Essas observações revelam as dificuldades para o controle da esquistossomose no estado de maior prevalência do Brasil.

\section{AGRADECIMENTOS}

Os autores agradecem aos acadêmicos Jamison Vieira de Matos Júnior e João Matheus Santos de Oliveira, pelo auxílio na coleta e armazenamento de dados; à MSc. Ana Denise Santana de Oliveira, pela interlocução com a Secretaria de Estado da Saúde para o fornecimento dos dados; e à Prof. Dra. Anna Klara Bohland, pelo suporte na análise estatística.

\section{CONFLITOS DE INTERESSE}

Os autores declaram não apresentar conflitos de interesses.

\section{CONTRIBUIÇÃO DOS AUTORES}

Todos os autores contribuíram com a idealização do texto, análise e interpretação dos dados, participando da redação e revisão do manuscrito e da aprovação da versão final publicada. Os autores se declaram responsáveis pelo conteúdo integral do artigo, garantindo sua precisão e integridade.

\section{REFERÊNCIAS}

1 Engels D, Chitsulo L, Montresor A, Savioli L. The global epidemiological situation of schistosomiasis and new approaches to control and research. Acta Trop. 2002 May;82(2): 139-46.

2 World Health Organization. Schistosomiasis: progress report 2001 - 2011, strategic plan 2012 - 2020. Geneva: WHO; 2013.

3 World Health Organization. Schistosomiasis [Internet]. Geneva: WHO; 2020 [cited 2020 Sep 18]. Available from: https://www.who.int/ news-room/fact-sheets/detail/schistosomiasis.
4 Ministério da Saúde (BR). Secretaria de Vigilância em Saúde. Coordenação-Geral de Desenvolvimento da Epidemiologia em Serviços. Guia de Vigilância em Saúde. 3. ed. Brasília: Ministério da Saúde; 2019.

5 Ministério da Saúde (BR). Secretaria de Vigilância em Saúde. Departamento de Vigilância das Doenças Transmissíveis. Coordenação Geral de Doenças em Eliminação. Vigilância da esquistossomose mansoni: diretrizes técnicas. 4. ed. Brasília: Ministério da Saúde; 2014. 
6 Ministério da Saúde (BR). Departamento de Informática do Sistema Único de Saúde. Programa de controle da esquistossomose [Internet]. Brasília: Ministério da Saúde; 2019 [citado 2019 nov 30]. Disponível em: http://tabnet.datasus.gov.br/cgi/ deftohtm.exe? sinan/pce/cnv/pcese.def.

7 Doumenge JP, Mott KE, Cheung G, Villenave D, Chapuis $O$, Perrine MF, et al. Atlas of the global distribution of schistosomiasis. Talence (FR): Centre d'Études de Géographie Tropicale; 1987.

8 Ministério da Saúde (BR). Secretaria de Vigilância em Saúde. Departamento de Vigilância das Doenças Transmissíveis. Coordenação Geral de Doenças Transmissíveis. Educação em saúde para o controle da esquistossomose. Brasília: Ministério da Saúde; 2018.

9 Coura JR, Mendonça MZG, Madruga JP. Tentativa de avaliação do Programa Especial de Controle da Esquistossomose (PECE) no Estado da Paraíba, Brasil. Rev Soc Bras Med Trop. 1987 abr-jun;20(2):67-76.

10 Ministério da Saúde (BR). Secretaria de Vigilância em Saúde. Departamento de Vigilância Epidemiológica. Guia de vigilância epidemiológica. 7. ed. Brasília: Ministério da Saúde; 2009. (Série A. Normas e Manuais Técnicos).

11 Katz N. Inquérito Nacional de prevalência da esquistossomose mansoni e geo-helmintoses. Belo Horizonte: Fiocruz/CPqRR; 2018. (Série esquistossomose, 17).

12 Menezes AP, Coura JR. Estudo seccional sobre esquistossomose mansônica no Município de Riachuelo, Estado de Sergipe. Rev Soc Bras Med Trop. $1980 \mathrm{dez} ; 13(1): 1-15$.

13 Coura JR, Conceição MJ, Menezes AP, Santos ML, Mendonça MZG. Morbidade da esquistossomose mansoni no Brasil: II - Estudo em quatro áreas de campo nos Estados de Minas Gerais, Sergipe e Paraíba. Mem Inst Oswaldo Cruz. 1983 jan-mar;78(1):1-11.

14 Carvalho EMF, Acioli MD, Branco MAF, Costa AM, Cesse EAP, Andrade AG, et al. Evolução da esquistossomose na Zona da Mata Sul de Pernambuco. Epidemiologia e situação atual: controle ou descontrole? Cad Saude Publica. 1998 out-dez; 14(4):787-95.

15 Martins-Melo FR, Pinheiro MCC, Ramos Jr AN, Alencar CH, Bezerra FSM, Heukelbach J. Trends in schistosomiasis-related mortality in Brazil, 20002011 . Int J Parasitol. 2014 Dec;44(14):1055-62.

16 Ministério da Saúde (BR). Secretaria de Vigilância em Saúde. Departamento de Vigilância em Doenças Transmissíveis. Coordenação Geral de Hanseníase e Doenças em Eliminação. Plano integrado de ações estratégicas de eliminação da hanseníase, filariose, esquistossomose e oncocercose como problema de saúde pública, tracoma como causa de cegueira e controle das geo-helmintíases: plano de ação 2011-2015. Brasília: Ministério da Saúde; 2012. (Série C. Projetos, programas e relatórios).
17 Nascimento GL, Pegado HM, Domingues ALC, Ximenes RAA, Itria A, Cruz LN, et al. The cost of a disease targeted for elimination in Brazil: the case of schistosomiasis mansoni. Mem Inst Oswaldo Cruz. 2019 Jan;114:e180347.

18 Barbosa CS, Souza ATOF, Leal Neto OB, Gomes ECS, Araujo KCGM, Guimarães RJPS et al. Turismo de risco para esquistossomose mansônica em Porto de Galinhas, Estado de Pernambuco, Brasil. Rev Pan-Amaz Saude. 2015 set;6(3):51-8.

19 Instituto Brasileiro de Geografia e Estatística. Cidades@: Sergipe [Internet]. Rio de Janeiro: IBGE; 2010 [citado 2019 nov 30]. Disponível em: https:// cidades.ibge.gov.br/brasil/se.

20 Secretaria de Estado da Saúde de Sergipe (BR). Esquistossomose: Sergipe possui 51 municípios endêmicos. Aracaju (SE): Secretaria de Estado da Saúde; 2017 [citado 2019 nov 30]. Disponível em: https://www.saude.se.gov.br/?p=15223.

21 Microsoft. Microsoft Excel 2016 [Internet]. Redmond (WA): Microsoft Corporation; 2015 [cited 2019 Nov 30]. Available from: https://www.microsoft.com/ pt-br/p/office-365-home/cfq7ttc0k5dm?icid=mscom marcom\&activetab $=$ pivot:overviewtab.

22 World Health Organization. Prevention and control of schistosomiasis and soil-transmitted helminthiasis: report of o WHO expert committee. Geneva: $\mathrm{WHO}$; 2002. (WHO technical report series; 912).

23 National Cancer Institute (USA). Joinpoint Regression Program. Version 4.7.0.0 [Internet]. Bethesda (MD): Surveillance Research Program; 2019 [cited 2019 Nov 30]. Available from: https:// surveillance.cancer.gov/joinpoint/.

24 Ayres M, Ayres Jr M, Ayres DL. BioEstat 5.0: aplicações estatísticas nas áreas das ciências biológicas e médicas. Belém (PA): Sociedade Civil Mamirauá; 2007.

25 Katz N, Peixoto SV. Análise crítica da estimativa do número de portadores de esquistossomose mansoni no Brasil. Rev Soc Bras Med Trop. 2000 mai-jun;33(3):303-8.

26 Rollemberg CV, Santos CMB, Silva MMBL, Souza AMB, Silva AM, Almeida JAP, et al. Aspectos epidemiológicos e distribuição geográfica da esquistossomose e geo-helmintos, no Estado de Sergipe, de acordo com os dados do Programa de Controle da Esquistossomose. Rev Soc Bras Med Trop. 2011 jan-fev;44(1):91-6.

27 Albuquerque MFPM, Souza WV, Araújo TVB, Braga MC, Miranda Filho DB, Ximenes RAA, et al. Epidemia de microcefalia e vírus Zika: a construção do conhecimento em epidemiologia. Cad Saude Publica. 2018 out;34(10):e00069018.

28 Alves JAB, Santos JR, Mendonça EN, Abud ACF, Nunes MS, Fakhouri $R$, et al. Epidemiological aspects of dengue in Aracaju, State of Sergipe, Brazil. Rev Soc Bras Med Trop. 2011 Nov-Dec;44(6):670-3. 
29 Rocha GCM, Souza JLS, Santos CS, Oliveira FES, Silva JÁ, Mendes MS, et al. Epidemiologia da dengue em Sergipe, entre 2009 e 2013. Cad Grad Cienc Biol Saude Unit. 2014 mar;2(1):103-9.

30 Ministério da Saúde (BR). Departamento de Informática do Sistema Único de Saúde. Sistema de Informação de Agravos de Notificação. Doenças e agravos de notificação - de 2007 em diante [Internet]. Brasília: Ministério da Saúde; 2019 [citado 2019 nov 30]. Disponível em: http://www2.datasus.gov.br/DATASUS/index. php? area $=0203 \&$ id $=29878153$.

31 Santos $A D$, Lima ACR, Santos MB, Alves JAB, Góes MAO, Nunes MAP, et al. Spatial analysis for the identification of risk areas for schistosomiasis mansoni in the State of Sergipe, Brazil, 2005-2014. Rev Soc Bras Med Trop. 2016 Sep-Oct;49(5):608-15.

32 Farias LMM, Resendes APC, Sabroza PC, Souza-Santos R. Análise preliminar do Sistema de Informação do Programa de Controle da Esquistossomose no período de 1999 a 2003. Cad Saude Publica. 2007 jan;23(1):235-9.

33 Silva LF, Nunes BEBR, Leal TC, Paiva JPS, Lemos AMS, Araújo LMM, et al. Schistosomiasis mansoni in the northeast region of Brazil: temporal modeling of positivity, hospitalization, and mortality rates. Rev Soc Bras Med Trop. 2019 Apr;52:e20180458.

34 Rocha TJM, Santos MCS, Lima MVM, Calheiros CML, Wanderley FS. Aspectos epidemiológicos e distribuição dos casos de infecção pelo Schistosoma mansoni em municípios do Estado de Alagoas, Brasil. Rev Pan-Amaz Saude. 2016 jun;7(2):27-32.

35 Coutinho AD, Silva ML, Gonçalves JF. Estudo epidemiológico da esquistossomose mansônica em áreas de irrigação do Nordeste brasileiro. Cad Saude Publica. 1992 jul-set;8(3):302-10.

36 Carmo EH, Barreto ML. Esquistossomose mansônica no estado da Bahia, Brasil: tendências históricas e medidas de controle. Cad Saude Publica. 1994 out-dez;10(4):425-39.

37 Tibiriçá SHC, Guimarães FB, Teixeira MTB. A esquistossomose mansoni no contexto da política de saúde brasileira. Cienc Saude Coletiva. $2011 ; 16$ supl 1:1375-81.

38 Organização das Nações Unidas. Programa das Nações Unidas para o Desenvolvimento. Atlas do Desenvolvimento Humano do Brasil. Percentual de pessoas em domicílios com abastecimento de água e esgotamento sanitário inadequados e IDHM [Internet]. Brasília: PNUD; 2010 [citado 2019 dez 2]. Disponível em: http://www.atlasbrasil.org. $\mathrm{br} / 2013 / \mathrm{pt} /$ consulta/.
39 Organização das Nações Unidas. Programa das Nações Unidas para o Desenvolvimento. Desenvolvimento humano para além das médias: 2017. Brasília: PNUD; 2017.

40 Silva JP, Ramos SB, Andrade MD. Análise multivariada da esquistossomose no estado de Minas Gerais: análise de componentes principais. ABCS Health Sci. 2018 ago;43(2):84-90.

41 Merchán-Hamann E. Diagnóstico macrorregional da situação das endemias de Regiões Norte e Nordeste. Inf Epidemiol Sus. 1997 jul-set;6(3):43-114.

42 Barbosa CS, Domingues ALC, Abath F, Montenegro SML, Guida U, Carneiro J, et al. Epidemia de esquistossomose aguda na praia de Porto de Galinhas, Pernambuco, Brasil. Cad Saude Publica 2001 mai-jun;17(3):725-8

43 Barbosa C, Araújo K, Antunes L, Favre T, Pieri O. Spatial distribution of schistosomiasis foci on Itamaracá Island, Pernambuco, Brazil. Mem Inst Oswaldo Cruz. 2004 Aug;99 Suppl 1:79-83.

44 Souza MAA, Barbosa VS, Wanderlei TNG, Barbosa CS. Criadouros de Biomphalaria, temporários e permanentes, em Jaboatão dos Guararapes, PE. Rev Soc Bras Med Trop. 2008 mai-jun;4 1 (3):252-6.

45 Leal Neto OB, Gomes ECS, Oliveira Jr FJM, Andrade R, Reis DL, Souza-Santos R, et al. Biological and environmental factors associated with risk of schistosomiasis mansoni transmission in Porto de Galinhas, Pernambuco State, Brazil. Cad Saude Publica. 2013 Feb;29(2):357-67.

46 Barbosa CS, Barbosa VS, Melo FL, Melo MSB, Bezerra L, Campos JV, et al. Casos autóctones de esquistossomose mansônica em crianças de Recife, PE. Rev Saude Publica. 2013 ago;47(4):684-90.

47 Correia EIS, Martinelli RP, Rocha H. Está desaparecendo a glomerulopatia da esquistossomose mansônica? Rev Soc Bras Med Trop. 1997 jul-ago;30(4):341-3.

48 Nascimento GL. Formas graves da esquistossomose mansoni: carga epidemiológica e custos no Brasil em 2010 [dissertação]. Brasília (DF): Universidade de Brasília, Faculdade de Medicina, Programa de Pós-Graduação em Medicina Tropical. 2013.73 p.

49 Ministério das Cidades (BR). Secretaria Nacional de Saneamento Básico. Plano Nacional de Saneamento Básico. Brasília: Ministério das Cidades; 2013. 